\title{
Artificial intelligence in education: The urgent need to prepare teachers for tomorrow's schools
}

Thierry Karsenti

Université de Montréal

(Canada)

doi:10.18162/fp.2019.a166

\section{HRONIQUE • Technologies en éducation}

\section{Introduction}

Two decades ago, no one had heard about Google, Facebook, YouTube, or Wikipedia. Today they are the most used digital tools on the planet. In this swiftly mutating world, digital technology exerts a tremendous influence on the economic, social, and cultural evolution of all societies. As new forms of technology continue to pervade our lives and captivate our youth, schools have no choice but to make room for them. At the same time, and in line with 21st century visions, countries expect their teachers and students to behave as informed and responsible digital citizens. Not to be left behind, Québec launched its Digital Action Plan for Education and Higher Education" in May 2018. It states that, "The shift to digital is a unique opportunity for the development and growth of Québec" (p. 3), and that, "digital technologies play a role in the educational success of our young people by offering them new ways to learn, communicate, share, create and collaborate: in short, by breathing new life into our schools" (p. 5). Moreover, these tools must be integrated effectively and used optimally in order to develop digital literacy and to foster informed and critical citizenship in the digital age. In addition, teachers need to be prepared for Québec's shift to digital: "Training and support for teachers and other school staff are extremely important, since they are the cornerstone of the action plan" (p. 5).

It is now 2018, and we can no longer afford to turn a deaf ear to these winds of change or turn our backs on the new avenues for education. Nor can we ignore the massive inroads that artificial intelligence (AI) has made into every corner of society. Although AI has not yet 
completely conquered the classroom, at least officially, it has certainly established a presence. Students and teachers are using AI every time they fire up a smart phone app or web browser, while perhaps unaware of the ethical implications. From now on, AI will be an integral part of our lives. Unfortunately, most school curricula and teacher training programs don't offer AI courses.

The objective of this column is to provide an overview of the educational uses of AI. This topic is far too broad to cover in a few pages, so we will narrow our focus to the importance of training teachers in the basics of AI for education. We can't ask all teachers to become AI experts, but they should at least be prepared to work in the schools of the future. At the same time, we must equip our young people with the tools they'll need to build tomorrow's society.

\section{Artificial intelligence: key concepts}

The aim here is not to provide an exhaustive description of how AI is used for education, but instead to define a few key terms.

\section{Artificial intelligence (AI)}

What is AI? In a nutshell, it's a branch of computer science that creates "intelligent" machines to work and react something like the human brain. Examples are computer programs (e.g., online platforms) and computerized machines (e.g., robots). Thanks to knowledge engineering and machine learning, these machines can process data, patterns, and models in order to perceive, reason, plan, solve problems, make predictions, and manipulate objects. One of the main advantages is that by automating complicated and time-consuming tasks, AI frees up time that would be spent on them. AI has many uses in the education field, as we shall see below.

\section{Big data}

Big data can be pictured as a digital ecosystem containing extremely large data sets that can be captured, managed, processed, and transferred. This is where AI comes in. AI uses algorithms to process huge amounts of data that regular computing techniques can't handle. AI is ideal for identifying trends and patterns and for making predictions. For example, the UTIFEN ${ }^{2}$ online platform connects 20,000 teachers, 7,000 teachers-in-training, and 1,500 teacher trainers to a certification program via their smart phones. The system responds to learner input, so individuals can learn according to their own pace and circumstances. The program covers eight to ten modules, each requiring about a hundred computer actions. This amounts to about 20,000,000 actions (or data units) that can be interpreted as patterns, or models. These models in turn provide insights into the learning pathways of those who complete the course and those who don't. In this way, AI enables the platform to "learn," and the outcome is that a wider range of students can earn a teaching certificate. 


\section{Algorithms}

AI is based on algorithms, which are basically sets of instructions that tell a computerized system, such as UTIFEN, how to handle data. Algorithms can be thought of as shortcuts to getting a computer to do something with data that it normally couldn't. An example is image recognition, as explained below.

\section{Machine learning / automated learning}

In machine learning, algorithms are tested and tuned so that they can treat data automatically to generate certain desired results, such as predictions and decisions. For example, the UTIFEN platform takes all the success and failure pathways of its users into account and then creates "ideal" intervention models for learner success. The platform then sends automated personalized reminders to users to help them get through the course. This type of machine learning does not use hand-coded software routines with specific sets of instructions. Instead, it is "trained" according to the best tested algorithms for specific problems. Machine learning is particularly suitable for image recognition. For example, leafsnap ${ }^{3}$ is a mobile app that uses visual recognition software to identify tree species from smart phone photos. It also provides detailed information about the trees. As schools continue to use tablets in classrooms, machine learning offers tremendous cognitive potential, far beyond the benefits for student motivation.

\section{Deep learning}
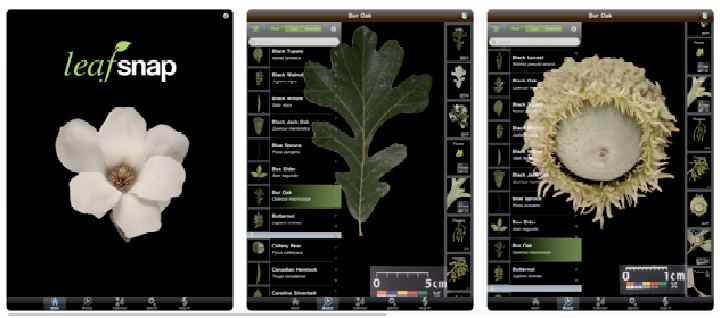
hierarchical training system where data are interpreted in terms of interconnections, layers of meaning, and propagation directions. To illustrate, a cat has a set of typical features: certain shapes for its head, fur, nose, ears, and so on. To recognize an image of a cat, a search engine such as Google interprets a vast number of the elements of these features, or inputs. These inputs are weighted for relevance and accuracy as the system advances through a series of interconnected data layers that grow in complexity and accuracy. Finally, the system makes a highly educated guess (akin to a human conclusion) that a certain image is indeed a cat. More interestingly, Google can recognize any cat as a cat. This kind of deep learning is very useful for making predictions. So, instead of manually coding software with specific instructions, the machine is given a set of training data and then tasked to sort through huge amounts of data on its own. For example, Facebook and Apple Photos build their training data sets from millions of accessed photos, from which they "learn" to identify people in photos. 


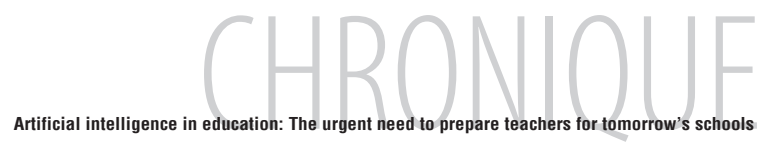

\section{Artificial intelligence for learning is here in Québec and the rest of Canada}

As we have seen, AI is already being used for learning. Teachers and students use smart phones and web browsers on a daily basis. For example, Duolingo, ${ }^{4}$ a language learning

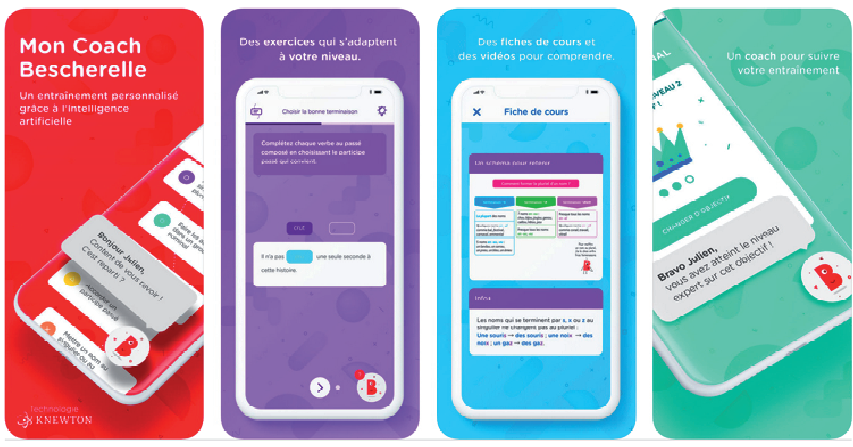
platform based on AI and voice recognition, is a very popular learning companion, with over 200 million users worldwide. Thousands of teachers use it to liven up their language classes, and these numbers are only expected to rise. Using AI, the app can track students' progress and provide personalized feedback and practice. Other language learning platforms such as $\mathrm{ELSA}^{5}$ (in English) offer personalized help with pronunciation skills.

From now on, universities will make increasing use of AI-based apps to detect plagiarism in students' work. For example, Turnitin ${ }^{6}$ recognizes degrees of plagiarism in students' work when they "turn it in." It shows the parts that are likely to have been plagiarized, the potential sources, and the percentages of these sources that have been plagiarized.

Adaptive learning is another important AI trend. In this case, AI is used to match students with learning pathways that are appropriate for their needs, learning style, and characteristics. The algorithm adapts in real time to each interaction with the student. Content can be added or removed and the difficulty level can be varied so that students can learn at their own pace. Major actors such as Pearson ${ }^{7}$ and $\mathrm{McGraw}-\mathrm{Hill}^{8}$ have invested heavily in this area, offering digital learning platforms that allow teachers to personalize and enrich their courses. The concept is simple: the learning pathway evolves continuously according to the learner's performance, needs, skills, interest level, and other characteristics.

Unfortunately, AI has made few inroads into Canadian and Québec education systems. The heavy costs are partly to blame. However, some promising applications are on the horizon, for example, Classcraft. ${ }^{9}$ Created in 2018, this online platform turns learning into a role-playing adventure. Teachers swear that it fosters teamwork, improves classroom behavior, and boosts engagement and motivation. As part of an ongoing project, Classcraft is drawing on data from over two million users to develop an AIassisted system that will make day-to-day classroom management easier and teaching more effective.

\section{6 positive impacts of Al on education}

Here are 26 contributions that artificial intelligence can make to education:

1. Personalized learning, for example, the UTIFEN project. This is arguably AI's greatest gift to education.

2. Greater academic success, again, as demonstrated by UTIFEN.

3. Automatic correction of certain kinds of schoolwork, which frees up teachers' time for other tasks. Unfortunately, the current thesis correction software (mostly available in English) leaves something to be desired. Despite the amazing progress made, the human touch remains essential. 
4. Ongoing student assessment: learners' experiences along the learning pathway are tracked in real time to accurately gauge skills acquisition over time.

5. Teachers can adjust their courses, to some extent. For example, Coursera ${ }^{10}$, a MOOC ${ }^{11}$ platform, tells teachers when too many students answer a question incorrectly or hand in inadequate work.

6. Intelligent tutoring platforms for distance learning. This is a growing trend, and combined with the rapid expansion of mobile technology, it opens up exciting opportunities for learners and educators alike.

7. New ways to interact with information. For example, Google adjusts our search results according to our geographic location or previous searches, generally without our knowledge. Amazon does the same when it suggests purchases in light of what we bought in the past. Siri, Apple's voice recognition assistant, adapts to individual voices, needs, and requests.

8. Educational feedback. For example, UTIFEN sends personalized texts to students as they follow their learning pathway. Not only is the feedback personalized, it's faster and more frequent, it allows automated grading, and it offers support and tailored recommendations.

9. Adapted teaching content, such as the digital bookshelves published by Pearson and McGrawHill.

10. Expanded opportunities for learners to communicate and collaborate with each other.

11. Greater interaction between learners and academic content. An example is the chatbot, an offspring of the original smart speakers like HomePod, Amazo Echo, and Google Home. A chatbot can recognize the user's language and simulate a real conversation.

12. Better teaching through facilitation rather than content transmission. But make no mistake: the teacher remains the star of the classroom, while AI plays a supporting role by handling complex digital tasks.

13. Homework assistance: students can do personalized homework that suits their academic skills and challenges. The online homework helper Allô prof, ${ }^{12}$ which has assisted students for over 20 years, would certainly benefit from AI.

14. More learning, because AI can personalize exercises to make learning more meaningful and enjoyable.

15. Immersive environments, or virtual reality. These highly interactive, three-dimensional virtual worlds encourage students to engage with course material. For example, the educational game Assassin's Creed lets students appreciate history as they "live" through vivid and detailed historical situations and carry out intriguing missions. ${ }^{13}$ Such enriched, interactive experiences have direct positive impacts on learning.

16. Dropout prevention: AI can gather student data and rapidly warn schools about those who are at risk for dropping out so they can receive appropriate support before matters deteriorate.

17. AI makes distance learning more accessible and appealing. People can learn anywhere, anytime, and programs can be made to measure. The language learning system Duolingo is an outstanding example. 


\section{CHROMIOLIE}

18. Learner autonomy, a key mission for educators.

19. Better classroom management. For example, a virtual experience like Classcraft engages students.

20. Extensive gamification potential, and gaming contributes directly to student engagement.

21. More efficient administrative management: newsletters, student absences, and so on can be handled quickly and easily.

22. To some extent, AI can detect learners' moods, which is useful for adjusting teaching practices.

23. Data collection, storage, and security: AI's cloud technology allows capturing, organizing, analyzing, and producing knowledge from vast amounts of data, while keeping them secure. This addresses both ethical and educational issues.

24. Students with special needs stand to particularly benefit from AI.

25. Automated tasks: much time that is normally spent on important education tasks can be taken over by AI systems.

26. Humanoid robots: although they will probably never replace real-life teachers, despite Hollywood fantasies, life-like robots will play an ever larger role in classrooms. They will act as teacher's assistants by performing complex and time-consuming tasks. ${ }^{14}$

\section{Conclusion: What kind of schools do we want in the future?}

Is there a need to prepare teachers-in-training to work with AI? Yes, for so many reasons. AI already has a heavy influence on individuals and societies, and we need to develop critical perspectives on AI issues. If teachers are trained in AI use, it will help prevent technology abuses. Importantly, for AI to make a real contribution to academic success, and for all students, the teacher's role remains as central as ever, perhaps more than ever. Because intelligent robots will transform tomorrow's workplaces, children should begin preparing for the new reality as early as primary school. The technology players must not be allowed to have the only say in all this. We have seen that AI has penetrated all the education spheres, in the form of intelligent books, web browsers, education apps, and learning platforms, to name a few. Yet "education" gets only one mention on the Wikipedia page devoted to "artificial intelligence." 15 The question therefore arises: what is our vision for the schools of the future? Will the technology giants be in sole charge of the ways that artificial intelligence is used for learning? Or will students and teachers be able to ask questions and provide clear, constructive, responsible, and ethical guidelines for how technology is used? The money-makers should not be allowed to decide our future without our say-so. The uses of AI must be carefully planned by the entire spectrum of education actors, starting with the teachers and learners. All this aligns with Québec's vision for its proposed international observatory on the societal impacts of artificial intelligence and digital technologies. According to Rémi Quirion, ${ }^{16}$ Chief Scientist of Québec, it will serve as an international hub for AI research, and it will demonstrate Québec's leadership in the ethically and socially responsible development of AI.

Many experts, including Yoshua Bengio at the Université de Montréal, ${ }^{17}$ worry about the risks of AI for society if the forward march of intelligent machines is left unchecked. But before we apply the breaks, we need to have some idea of how it works and what it does. It would also be wise to bring in advisors and experts who are not technology stakeholders. From now on, we'll have to live with AI, so isn't it about time that we get our teachers ready for it? 
In terms of the benefits for education, AI is neither a panacea nor the Holy Grail. Instead, it's a tool with tremendous potential, and one that we must learn to use to its full advantage. One major challenge is to find the right balance between the time-honoured teaching practices that have been handed down for centuries and the new possibilities afforded by AI. Moreover, we should not limit ourselves to a simple utilitarian vision of AI. Instead, we could try to imagine how learning can be nurtured and transformed. Ultimately, $\mathrm{AI}$ is not just good for school: it's also good for creating greater understanding and respect between all people.

\section{Notes}

1 Ministère de l'Éducation et de l'Enseignement supérieur (2018). Plan d'action numérique en éducation et en enseignement supérieur. Québec : Gouvernement du Québec.

2 UTIFEN: Use of Technology for Mobile Teacher Training in Niger. http://www.utifen.org:81/wp/

3 https://itunes.apple.com/us/app/leafsnap-for-ipad/id433522683?mt=8

4 https://www.duolingo.com/

5 https://itunes.apple.com/us/app/elsa-speak-accent-reduction/id1083804886?mt=8\&ign-mpt=uo\%3D4

6 https://www.turnitin.com/

7 http://researchnetwork.pearson.com/digital-data-analytics-and-adaptive-learning

8 http://www.learnsmartadvantage.com/about/

9 https://www.classcraft.com/fr/

10 https://www.coursera.org/

11 Massive Open Online Courses

12 http://alloprof.qc.ca

13 See Karsenti, T. ; Bugmann, J. et Parent, S. (2018). Apprendre l'histoire avec le jeu Assassin's Creed ? Une étude exploratoire menée auprès de 329 élèves du secondaire. Montréal : CRIFPE.

14 See, e.g., Karsenti, T., Bugmann, J., \& Gros, P-P. (2017). Using Humanoid Robots to Support Students with Autism Spectrum Disorder. Formation et profession, 25(3) 134-136. http://dx.doi.org/10.18162/fp.2017.a135

15 https://en.wikipedia.org/wiki/Artificial intelligence

16 Government of Québec, March 29,2018. Québec lays the groundwork for a world observatory on the social impacts of artificial intelligence and digital technologies. http://www.scientifique-en-chef.gouv.qc.ca/en/nouvelles/quebec-jette-bases-dun-observatoire-

17 Interview with Yoshua Bengio (2016). Will Machines Eliminate Us ? MIT Technology Review. https://www.technologyreview.com/s/546301/will-machines-eliminate-us/.

\section{Pour citer cet article}

Karsenti, T., (2019). Artificial intelligence in education: The urgent need to prepare teachers for tomorrow's schools. Formation et profession, 27(1), 105-111. http://dx.doi.org/10.18162/fp.2018.a166 